\title{
Erratum: Fixed-density boundary conditions in overdamped Langevin simulations of diffusion in channels [Phys. Rev. E 98, 013302 (2018)]
}

\author{
L. Ramírez-Piscina $\odot$
}

Q (Received 20 July 2020; published 31 July 2020)

DOI: 10.1103/PhysRevE.102.019904

There were misprints in Eqs. (17) and (19) of my paper. The correct Eq. (17) should read

$$
x \simeq f \Delta t+\left(-4 \Delta t \frac{k_{B} T}{\gamma} \ln [2 \sqrt{\pi} q(a)(1-\chi)]\right)^{1 / 2}
$$

and the correct Eq. (19) should read

$$
x=f \Delta t+\left(4 \Delta t \frac{k_{B} T}{\gamma}\right)^{1 / 2} y .
$$

These misprints do not impact the presented results and conclusions; all simulations in the paper have been performed by using the correct equations.

I would like to thank Josep Bataller, Guillermo Villanueva, and Ce Xu Zheng for reporting numerical inconsistencies when using the published equations. 